\title{
EFEITO DO LIGNOSSULFONATO DE CÁLCIO E MAGNÉSIO NA RESISTÊNCIA MECÂNICA E CONSUMO DE ENERGIA NA FABRICAÇÃO DE ALIMENTO EXTRUSADO PARA CÃES
}

\author{
THIAGO DO NASCIMENTO ${ }^{1}$, ANTONIO C. DE. O. FERRAZ ${ }^{2}$, JOÃO D. BIAGI ${ }^{3}$
}

RESUMO: Utiliza-se a extrusão termoplástica na produção de alimento animal para obter extrusados que se mantenham íntegros, pois sua desintegração resulta em oneroso reprocessamento. Os objetivos do presente trabalho foram avaliar o efeito da adição de lignossulfonato de cálcio e magnésio, nas concentrações de 0,$5 ; 1,0 ; 1,5 ; 2,0$ e 4,0\%, em massa, em formulação genérica para cães adultos em manutenção, na resistência mecânica do extrusado e medir o consumo de energia elétrica da extrusora, em escala industrial. Não houve diferenças na resistência mecânica do extrusado, quando avaliado através de penetração com ponteira cilíndrica. Porém, utilizando a compressão entre pratos planos rígidos e paralelos, discriminou-se a resistência dos extrusados que continham lignossulfonato em sua formulação daqueles da testemunha. A adição do lignossulfonato não afetou a durabilidade do extrusado. O tempo de processamento diminuiu com o aumento da quantidade de lignossulfonato presente na formulação da ração.

PALAVRAS-CHAVE: energia elétrica, extrusão, ração, resistência mecânica.

\section{EFFECT OF CALCIUM AND MAGNESIUM LIGNOSULFONATE IN THE MECHANICAL RESISTANCE AND ENERGY CONSUMPTION DURING PROCESSING OF DOG EXTRUDED FOOD}

\begin{abstract}
Thermoplastic extrusion of animal food is used to obtain integral extrudates since disintegration results in costly reprocessing. In this paper we outline the calcium and magnesium lignosulfonate addition effect on extrudate mechanical resistance for adult dog maintenance generic formulation in mass concentrations of $0.5 ; 1.0 ; 1.5 ; 2.0$ and $4.0 \%$. And also measure extruder's electrical energy consumption at industrial scale. Results showed no differences in extrudate mechanical resistance when penetration with a cylindrical probe was performed. However, compression of rigid flat parallel plates showed differences between lignosulfonate extrudates and control. The extrudate durability was not affected by lignosulfonate addition. Processing time decreased as the lignosulfonate amount increased.
\end{abstract}

KEYWORDS: animal feed, extrusion, extrudates, electrical energy.

\section{INTRODUÇÃO}

Alimentos industrializados, completos e balanceados para cães e gatos (Pet Food) são práticos, econômicos e eficazes, satisfazendo às exigências nutricionais destes animais. A aceitação destes produtos pelo mercado aumentou intensamente quando os alimentos passaram a ser extrusados e não mais peletizados, pois com este novo processamento atingiram-se melhores resultados em digestibilidade e palatabilidade.

A produção nacional em 2012 atingiu 63 milhões de toneladas, sendo que o segmento de animais de companhia aumentou sua produção em 4\% com relação a 2011, resultando num faturamento no varejo de R\$ 9,5 bilhões (SINDIRAÇÕES, 2013). O aumento da população de cães

\footnotetext{
${ }^{1}$ Zootecnista, Mestre em Engenharia Agrícola, Campinas - SP, thiago.n@terra.com.br

${ }^{2}$ Eng ${ }^{0}$ Agrícola, Prof. Doutor, Faculdade de Engenharia Agrícola - UNICAMP, Campinas - SP, carlos@feagri.unicamp.br

${ }^{3}$ Eng ${ }^{\underline{0}}$ Agrícola, Prof. Doutor, Faculdade de Engenharia Agrícola - UNICAMP, Campinas - SP, biagi@feagri.unicamp.br

Recebido pelo Conselho Editorial em: 7-6-2011

Aprovado pelo Conselho Editorial em: 28-1-2014
} 
e gatos, decorrente da necessidade de companhia e proteção das pessoas, favorece o fortalecimento deste segmento. $\mathrm{O}$ setor cresce em volume e torna-se também mais exigente quanto à qualidade.

Com movimentação financeira expressiva e mercado promissor, o estudo dos processos envolvidos na produção destes tipos de alimentos, assim como a utilização de aditivos que melhorem a qualidade, é de fundamental importância para o desenvolvimento da indústria nacional (SINDIRAÇÕES, 2011).

Um dos objetivos importante na produção de alimento animal, tanto para a indústria como para os animais, é a manutenção do extrusado da ração íntegro após a fabricação. Na indústria, a desintegração do produto produz uma quantidade de farelo, também denominado de finos, que resulta em reprocessamento indesejável, com consequente aumento de custos. Para os animais, o extrusado íntegro evita a segregação de nutrientes, garantindo um alimento balanceado. Quando o extrusado não apresenta adequada resistência mecânica à abrasão e à compressão, a utilização de aditivos aglutinantes é uma opção para melhorar essas características. Dos aditivos de maior utilização pela indústria de alimentação animal para este fim, estão os lignossulfonatos, subprodutos da indústria da celulose (KHITRIN et al., 2012). Possuem propriedades aglomerantes e emulsificantes, e sua adição durante a produção de alimento animal é destinada à formação de peletes com melhor qualidade, isto é, mais resistentes mecanicamente devido ao seu poder aglutinante (ACAR et al., 1991). São utilizados também como adsorventes (KHITRIN et al., 2012) e como aglomerantes em diversas aplicações, tais como na peletização de resíduos de madeira (TARASOV et al., 2013) e estruturas de alvenaria (MASKELL et al., 2012). Porém, as informações sobre o efeito do lignossulfonato, quando utilizado em formulações extrusadas, são muito limitadas (MELBAR, 2000).

A peletização e a extrusão termoplástica são processos bem distintos, pois neste último a mistura farelada é cozida com adição de água e vapor, podendo também receber aditivos, tais como óleo, gordura, carne, produtos à base de peixe, conservantes, entre outros. Na extrusão, materiais úmidos, expansíveis, amiláceos, proteicos, fibrosos e gordurosos são plastificados em um duto através da combinação de teor de água, pressão, calor e cisalhamento mecânico, resultando em aumento da temperatura da massa na extrusora, seguido de uma expansão exotérmica (FRAIHA, 2008). As propriedades físico-químicas, propriedades reológicas e características microbiológicas das matérias-primas são alteradas ao longo da extrusão (FRAIHA et al., 2010; KALIYAN \& MOREY, 2009; ROKEY et al., 2010), que atualmente não são perfeitamente entendidas, em grande parte, devido à dificuldade de instalação de sensores precisos e reprodutíveis para avaliar o comportamento da massa na extrusora. Comumente, na indústria de alimentação animal, a extrusão ainda é controlada de forma manual. A extrusão termoplástica para a produção de alimentos para animais de estimação, assim como para as demais aplicações na indústria alimentícia humana, é uma das operações unitárias mais empregadas atualmente, que pode ser avaliada como uma combinação entre transporte mistura compressão, cozimento e expansão de um grupo de matérias-primas (FRAIHA, 2008).

Devido à não existência de estudos na fabricação industrial de alimentos para cães utilizando extrusão termoplástica com adição de lignossulfonato de cálcio e magnésio, neste trabalho, foi o avaliado efeito de várias quantidades desse aditivo, em formulação comercial e escala industrial, na resistência mecânica, estabilidade do extrusado e consumo de energia elétrica pela extrusora.

\section{MATERIAL E MÉTODOS}

\section{Locais dos experimentos}

O processamento foi realizado na planta industrial de fabricante de alimentos para animais, localizada no município de Pitangueiras - SP, e as análises nos Laboratórios de Tecnologia PósColheita e Propriedades Mecânicas dos Materiais Biológicos, ambos pertencentes à Faculdade de Engenharia Agrícola, FEAGRI, Universidade Estadual de Campinas, UNICAMP, Campinas - SP. 


\section{Caracterização do material}

Foram utilizadas duas formulações balanceadas, denominadas formulação 1 e formulação 2, sendo a primeira destinada à manutenção para cães adultos e a segunda de crescimento para filhotes, cujos níveis de garantia de suas formulações e respectivas composições são apresentadas nas Tabelas 1 e 2. A formulação 1 foi utilizada para a avaliação das características mecânicas do extrusado, enquanto a formulação 2, para as medições do consumo de energia elétrica. Os níveis de garantia do lignossulfonato de cálcio e magnésio utilizados como aglutinantes são apresentados na Tabela 3.

TABELA 1. Níveis de garantia (\%) para as formulações 1 e 2 . Warranty level (\%) of formulations 1 and 2.

\begin{tabular}{cccc}
\hline Nutrientes & Limite (\%) & Formulação 1 & Formulação 2 \\
\hline Teor de água & Máx. & 12,00 & 12,00 \\
Proteína Bruta & Mín. & 18,00 & 25,00 \\
Extrato Etéreo & Mín. & 4,50 & 7,00 \\
Fibra Bruta & Máx. & 6,59 & 5,00 \\
Cálcio & Máx. & 2,50 & 2,00 \\
Fósforo & Mín. & 0,45 & 0,90 \\
\hline
\end{tabular}

TABELA 2. Composição (\%) das formulações 1 e 2. Compositions (\%) of formulations 1 and 2.

\begin{tabular}{|c|c|c|}
\hline \multirow{2}{*}{ Ingredientes } & \multicolumn{2}{|c|}{ Formulação } \\
\hline & 1 & 2 \\
\hline Aditivos + suplemento mineral e vitamínico & 1 & 1 \\
\hline 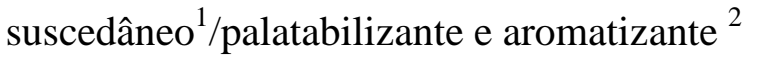 & & 1,2 \\
\hline Calcário & 0,4 & 1,4 \\
\hline Caulim amarelo & 1,5 & \\
\hline Farelo de casca de arroz & 0,5 & \\
\hline Farelo de soja $45 \%$ & & 20 \\
\hline Farelo de trigo & 45 & 29 \\
\hline Farinha de carne $45 \%$ & 19 & 9 \\
\hline Farinha de penas & & 3 \\
\hline Farinha de vísceras de aves & & 5 \\
\hline Hidrolisado de fígado & 0,1 & 2 \\
\hline Lactose $99 \%$ & & 0,8 \\
\hline Levedura de cerveja & & 0,5 \\
\hline Milho & 10 & 24 \\
\hline Óleo de frango & 2 & 2 \\
\hline Plasma sanguíneo & & 0,1 \\
\hline Sal comum & 0,5 & 1 \\
\hline Sorgo & 20 & \\
\hline
\end{tabular}


TABELA 3. Níveis de garantia do lignossulfonato de cálcio e magnésio. Warranty levels for calcium and magnesium lignosulfonate.

\begin{tabular}{lr}
\hline Nutrientes & \\
\hline Açúcares redutores totais & $22 \%$ \\
Cálcio (máximo) & $2,4 \%$ \\
Energia metabolizável & $2.280 \mathrm{kcal}$ \\
Magnésio (máximo) & $2,2 \%$ \\
Matéria mineral a $800{ }^{\circ} \mathrm{C}$ (máximo) & $10 \%$ \\
Nutrientes digestíveis totais (N.D.T.) & $63 \%$ \\
Teor de água (máximo) & $8 \%$ \\
\hline
\end{tabular}

\section{Parâmetros do processamento}

Para a produção da farelada, utilizou-se de peneiras com malha de 1,2 mm e $1 \mathrm{~mm}$, para beneficiar as formulações 1 e 2, respectivamente. Utilizou-se de uma extrusora FERRAZ (modelo E-200 com motor de 147,1 kW (200 cv), de helicoide simples, com capacidade nominal de 6,5 $\mathrm{t} \mathrm{h}^{-1}$ à rotação do helicoide de 4,67 Hz (280 rpm)). As medidas do diâmetro externo e do comprimento do helicoide são de 200 e 3.300 mm, respectivamente, e o diâmetro dos orifícios da matriz, de $10 \mathrm{~mm}$. A temperatura da mistura foi elevada mediante injeção de vapor d'água durante a etapa do condicionamento (Figura $1 \mathrm{~A}$ ), mantendo-se em $90{ }^{\circ} \mathrm{C}$ nas zonas de alimentação, cozimento e formatação (Figura 1 B, C, D). Para a caracterização da massa, foi realizada a determinação do teor de água pelo método número 2 do COMPÊNDIO BRASILEIRO DE ALIMENTAÇÃO ANIMAL (SINDIRAÇÕES, 2009), com amostras extraídas do condicionador.

Após a mistura dos ingredientes em misturador horizontal, $2.000 \mathrm{~kg}$ por tratamento, por 5 minutos, a mesma foi condicionada e, em seguida, extrusada (Figura 1), seguindo-se de secagem com ar aquecido, em secador horizontal de esteira, por 22 minutos, a $140{ }^{\circ} \mathrm{C}$. O mesmo conjunto de equipamentos, tratamentos e condições de mistura e secagem foram utilizados para ambas as formulações, que receberam processamento contínuo, para ambas as formulações, sem interrupção no carregamento das distintas misturas para cada tratamento.

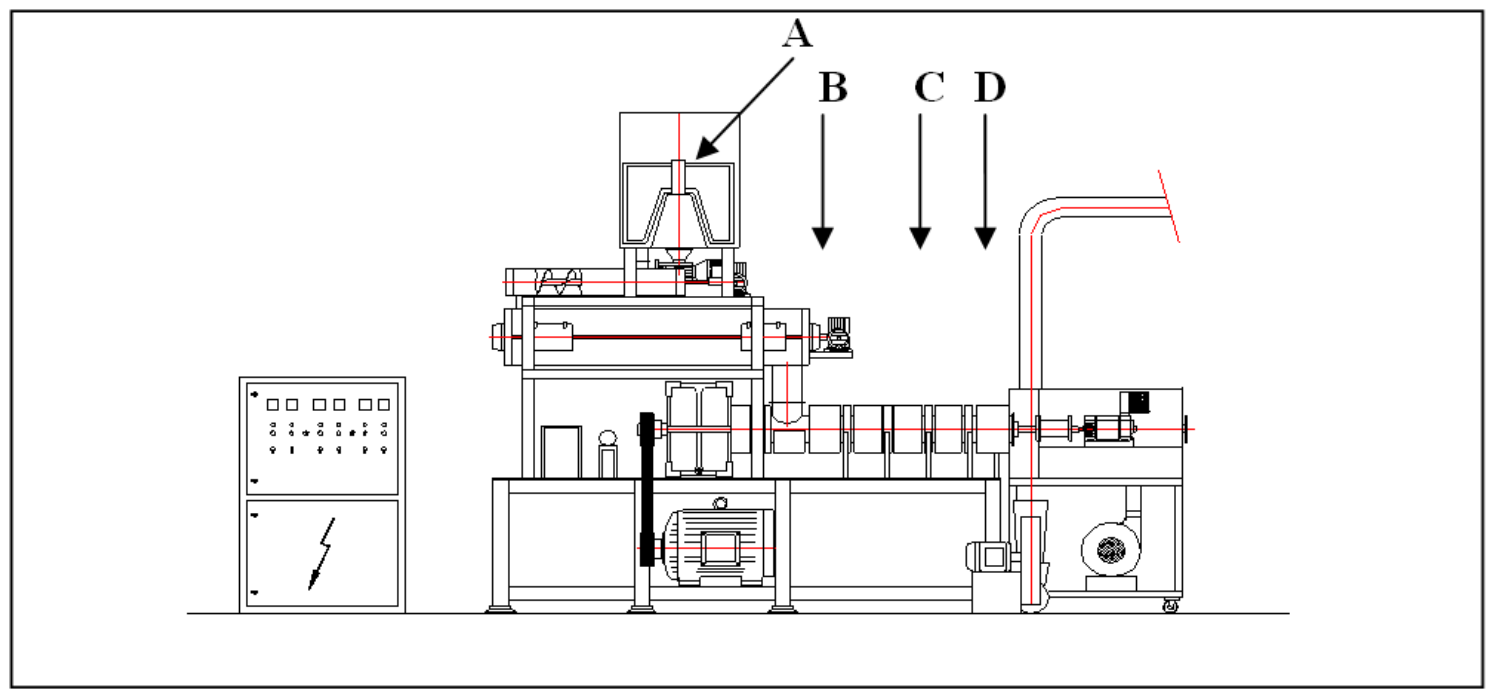

FIGURA 1. Representação esquemática da extrusora, mostrando: condicionador (A), zonas de alimentação (B), cozimento (C) e formatação (D). Adaptado de TERRA, 2000. Schematic representation of extruding machine: conditioner (A), input gate (B), cooking zone (C) and shaping stage (D). Adapted from TERRA, 2000. 


\section{Tratamentos}

Mantendo-se os mesmos procedimentos para a mistura dos ingredientes, extrusão e secagem, para as formulações 1 e 2, variou-se a concentração de lignossulfonato de cálcio e magnésio, em massa, resultando os seguintes tratamentos: Tratamento 1 (T1): formulação sem adição de lignossulfonato de cálcio e magnésio (testemunha); Tratamentos 2; 3; 4; 5 (T2, T3, T4, T5,): formulação com adição de 0,5; 1,0; 1,5 e 2,0\% de lignossulfonato de cálcio e magnésio, respectivamente; Tratamento 6 (T6): formulação com adição de 4,0\% de lignossulfonato de cálcio e magnésio, somente para a formulação 2 .

\section{Delineamento experimental}

O delineamento experimental foi o de mistura de dois componentes (CHARNET et al., 2008), sendo o primeiro o lignossulfonato de cálcio e magnésio, e o segundo, os demais ingredientes, considerados variáveis dependentes. Para a formulação 1, foram realizadas quatro repetições por tratamento, totalizando 20 misturas de material a extrusar, sendo a ordem do processamento totalmente casualizada. Nos experimentos utilizando a formulação 2, foram processados uma única vez, na seguinte ordem, os tratamentos $2 ; 3 ; 4 ; 5 ; 6$ e 1 . Os diversos tratamentos foram avaliados por meio da análise de variância e comparação de médias com o auxílio do software de análises estatísticas STATGRAPHICS Plus for Windows 4.1 (STATPOINT, 1999).

\section{Coleta do extrusado}

Logo após a extrusão, para a formulação 1, foram coletadas quatro amostras por tratamento, sendo as coletas realizadas na saída do secador, aos 7; 14; 21 e 28 minutos de processamento. Estas amostras foram acondicionadas em sacos plásticos de polietileno, com $10 \mu \mathrm{m}$ de espessura, e transportadas à Faculdade de Engenharia Agrícola da Unicamp e armazenadas em câmara fria a $17{ }^{\circ} \mathrm{C}$. Para realizar as avaliações do extrusado após a secagem, as quatro amostras de cada repetição foram misturadas para constituírem uma única amostra homogeneizada. Para a formulação 2, foram extraídas três amostras por tratamento na saída do secador, aos 10; 20 e 30 minutos de secagem, seguidas de procedimento semelhante ao utilizado para a formulação 1.

\section{Consumo de energia elétrica}

Utilizando-se de um medidor registrador eletrônico trifásico de grandezas elétricas, com memória de massa (ESB, modelo SAGA 4000), ligado ao painel elétrico de alimentação do motor da extrusora, os dados foram coletados automaticamente, em intervalos de 15 segundos, registrando-se o consumo de energia elétrica e a potência útil do motor da extrusora, de maneira semelhante ao utilizado por GONZÁLEZ et al. (2013).

\section{Tempo de duração da extrusão}

A medida do tempo de processamento de cada lote de $2.000 \mathrm{~kg}$ foi realizada, utilizando-se de cronômetro digital a partir do instante da descarga do farelado no silo de alimentação da extrusora. O enchimento deste silo foi realizado toda vez que o nível da mistura atingia o ponto mais baixo da janela de inspeção transparente do silo, quando ainda restavam, aproximadamente, $200 \mathrm{~kg}$ de mistura anterior. Nesse nível, ocorria a liberação da mistura do tratamento subsequente, enchendo o silo novamente. A partir desse instante, estimou-se, baseado na produção da extrusora, serem necessários 3,5 minutos até que a antiga mistura se esgotasse. A soma do tempo medido entre descargas com os 3,5 minutos adicionais foi o tempo considerado de processamento para cada repetição. Os valores de tempo de processamento foram correlacionados com valores de energia (CHARNET et al., 2008).

\section{Ensaios mecânicos}

Produtos destinados à alimentação animal são caracterizados mecanicamente pela resistência e durabilidade (resistência à abrasão e fragmentação). A resistência mecânica de material particulado pode ser avaliada pela resistência de uma porção de suas partículas, utilizando-se de células de cisalhamento, tal como a de Kramer, ou pela resistência individual de cada partícula 
(THOMAS et al., 1996). Devido à inexistência de procedimento normatizado para as medidas de resistência de extrusados, decidiu-se utilizar dois tipos de procedimentos: ensaios de penetração com ponteira cilíndrica e compressão entre pratos planos e paralelos, de naturezas distintas, buscando identificar aquele de maior potencial discriminatório. Para tal, utilizou-se de máquina universal de ensaios ou texturômetro ("Texture Analyzer”, LLOYD INSTRUMENTS LTD, modelo TA 500), equipada com software de aquisição de dados (NEXYGEN 3.0), célula de carga com capacidade máxima de $500 \mathrm{~N}$, e os ensaios foram realizados à taxa de deformação de 0,6 mm s${ }^{-1}$ Os valores de força e deformação foram coletados a uma frequência de $50 \mathrm{~Hz}$ e armazenados automaticamente. Foram utilizadas 25 unidades do extrusado por repetição, para cada tratamento. Ambos os procedimentos foram analisados quanto à variabilidade das medidas (coeficiente de variação) e potencial de discriminação entre tratamentos (comparação de médias).

Os ensaios de durabilidade seguiram procedimento normatizado e avaliam o efeito da manipulação do produto quantificando a fragmentação das partículas e formação de finos. Ambos os tipos de ensaios, de resistência e durabilidade, são descritos em detalhes a seguir.

\section{Ensaio de penetração}

Cada extrusado foi colocado sobre a superfície plana do prato inferior da Máquina Universal, na posição de repouso mais estável, e a ponteira cilíndrica, de 1,3 $\mathrm{mm}$ de diâmetro e ponta hemisférica, concordante com o corpo cilíndrico, foi baixada vagarosamente até tocar levemente a região central do extrusado. Nesse instante, inseriu-se no software o valor da altura do extrusado, medido pela própria máquina universal, realizando-se a penetração até 30\% desta. A partir da curva força-deformação obtida calculou-se a energia de deformação até 30\% de penetração. O valor da energia de deformação assim calculado foi considerado o índice de resistência do extrusado.

\section{Ensaios de compressão}

Para os ensaios de compressão entre pratos planos rígidos e paralelos, utilizou-se da mesma máquina universal, colocando-se o extrusado sobre o prato plano inferior, em sua posição mais estável de repouso, deformando-o até $30 \%$ de sua altura original. Semelhantemente ao ensaio anterior, o índice de resistência foi o valor da energia de deformação, comparando-se os tratamentos pelos valores médios obtidos.

Para ambos os ensaios de penetração e de compressão, a energia de deformação foi calculada pela área sob a curva força-deformação calculada por integração numérica (método dos trapézios).

Os dois procedimentos foram comparados quanto à variabilidade das medidas, avaliada pelo coeficiente de variação (WU \& ABBOT, 2002), visando a obter um procedimento com menor variabilidade e maior potencial de discriminação. Os valores médios foram comparados, utilizandose de análise de variância e dos testes LSD, Student-Norman_Keuls e Duncan (STATPOINT, 1999).

\section{Ensaio de durabilidade}

A determinação do índice de durabilidade foi realizada para as amostras retiradas na saída do secador. Esta análise evidencia os efeitos da manipulação do produto e foi realizada pelo método da ASAE Standard (1994): ASAE S269.4. As amostras de $500 \mathrm{~g}$ foram colocadas na caixa metálica, acoplada à castanha do torno (Nardini, modelo Nodus) e rotacionada por 10 minutos, a 5,3 rad s${ }^{-1}$ (150 rpm). Após este período, as amostras foram retiradas da caixa e peneiradas por 5 minutos (PRODUTEST, modelo “T”, com peneira Granutest ${ }^{\circledR}$ de 9,52 mm de abertura). Ao final desta etapa, foram pesados os extrusados restantes na pereira. A durabilidade foi expressa, em porcentagem da massa da fração retida na peneira, relativa à massa inicial da amostra. Foram ensaiadas quatro amostras por tratamento.

A caixa metálica utilizada foi projetada e construída segundo a norma citada. 


\section{RESULTADOS E DISCUSSÃO}

\section{Teor de água da mistura no condicionador}

O valor médio do teor de água entre os tratamentos foi de 25,97\%, muito próximo do valor de $26 \%$ pretendido para a mistura no pré-condicionador. Na Tabela 4, apresentam-se, para os tratamentos considerados, os valores médios do teor de água da mistura na saída do condicionador, assim como seus respectivos desvios-padrão e coeficientes de variação.

Verifica-se que esses valores são próximos e apresentam baixo coeficiente de variação, indicando processamento uniforme no condicionador.

TABELA 4. Valores médios do teor de água das misturas na saída do pré-condicionador, para formulação 1, com desvios-padrão (D.P.) e coeficientes de variação (C.V.), para cada tratamento. Average moisture content, standard deviation (D.P.) and coefficient of variation (C.V.) from pre-conditioner discharge for each treatment.

\begin{tabular}{cccccc}
\hline \multicolumn{5}{c}{ Tratamento } \\
\hline Média (\%) & 1 & 2 & 3 & 4 & 5 \\
D.P. (\%) & 25,99 & 26,22 & 25,59 & 26,25 & 25,84 \\
C.V. (\%) & 0,7123 & 0,6860 & 0,8245 & 0,6066 & 0,6660 \\
\hline
\end{tabular}

\section{Ensaio de penetração}

As curvas força-deformação específica obtidas dos ensaios de penetração apresentaram dois trechos distintos. No primeiro, até aproximadamente $15 \%$ da deformação específica, os valores de força oscilaram ligeiramente e apresentaram inclinação acentuada e, no segundo trecho, a variação da força com a deformação foi discreta, assemelhando-se a um patamar (Figura 2a). Esse comportamento na penetração sugere a existência de uma camada externa mais resistente, provavelmente com maior densidade, resultante da interação com as superfícies da matriz da extrusora, caracterizada por interações com maiores valores de atrito e temperatura, resultando em tratamento diferenciado daquela porção de material localizada no interior do extrusado. Os efeitos da secagem também estão presentes, uma vez que a camada externa perde água mais rapidamente que o interior do extrusado devido às altas temperaturas praticadas durante a secagem.

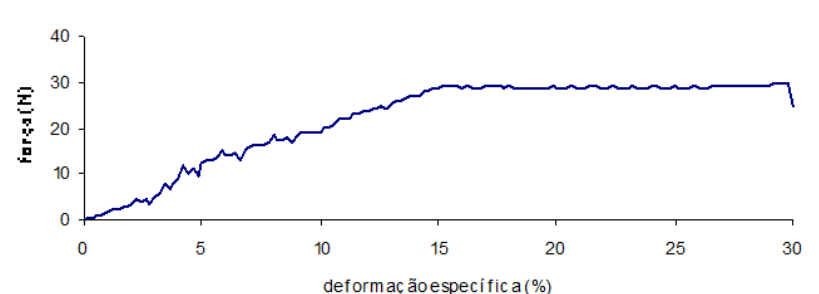

a

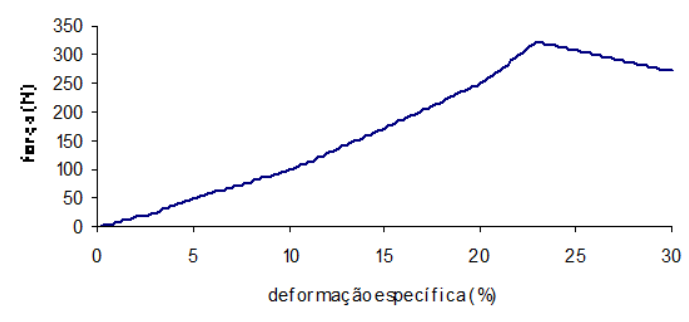

$\mathrm{b}$

FIGURA 2. a) Curva característica força-deformação específica obtida nos ensaios de penetração, utilizando ponteira cilíndrica de 1,3 mm de diâmetro, e deformação de 30\% da altura inicial; b) Curva características de força-deformação obtida nos ensaios de compressão entre pratos planos rígidos e paralelos com deformação de $30 \%$ da altura inicial. a) Characteristic force-deformation curve obtained from penetration test, up to $30 \%$ of initial specimen height, using a $1.3 \mathrm{~mm}$ diameter cylindrical probe; b) Characteristic force-deformation curve obtained from compression test, up to $30 \%$ of initial specimen height, using parallel and rigid plates. 
Os resultados dos ensaios de penetração (Tabela 5), utilizando-se de 100 extrusados por tratamento, apresentaram média geral do valor da energia de deformação de 0,0492 J. A análise de variância $(\mathrm{p}<0,05)$ para valores médios da energia de deformação não apresentou diferenças significativas entre os tratamentos.

Observa-se que os valores médios dos índices de resistência à penetração baseados na energia de deformação, apesar de apresentarem coeficientes de variação dentro do aceitável para materiais biológicos, próximos de 15\%, não discriminaram os diversos tratamentos.

TABELA 5. Valores médios do índice de resistência à penetração(J), para formulação 1, com respectivos desvios-padrão (D.P.) e coeficientes de variação (C.V.). Average, standard deviation (D.P.) and coefficient of variation (C.V.) of the penetration resistance index $(\mathrm{J})$, for formulation 1.

\begin{tabular}{cccccc}
\hline & \multicolumn{5}{c}{ Tratamento } \\
& 1 & 2 & 3 & 4 & 5 \\
\hline Média (J)* & 0,0508 & 0,0492 & 0,0491 & 0,0479 & 0,0488 \\
D.P. (J) & 0,0076 & 0,0075 & 0,0079 & 0,0081 & 0,0089 \\
C.V. (\%) & 15,02 & 15,31 & 16,15 & 16,90 & 18,25 \\
\hline
\end{tabular}

*para 100 repetições.

\section{Ensaios de compressão entre pratos planos, rígidos e paralelos}

As curvas força-deformação obtidas nos ensaios foram suaves, com crescimento monotônico até, aproximadamente, $23 \%$ de deformação ou a partir da força máxima, mudando de comportamento a partir desse valor. Muito embora a força máxima não seja um bom índice de resistência (FERRAZ \& HONÓRIO, 2008), valores entre 17,4 e 27,1 N foram obtidos para extrusados para peixes (SITAULA, 2011), que estão bem abaixo dos encontrados para a formulação utilizada. Na Figura 2b, ilustra-se, caracteristicamente, a relação entre a força e a deformação durante a compressão de um espécime.

Os valores médios da energia de deformação, até 30\% da altura inicial do espécime, obtidos para cada tratamento, assim como seus desvios-padrão e coeficientes de variação, são apresentados na Tabela 6. O valor médio de energia de deformação foi de $0,3256 \mathrm{~J}$, bastante superior aos valores obtidos com a ponteira. Para os tratamentos com adição de lignossulfonato, houve aumento significativo da resistência à compressão quando comparados ao tratamento 1. Os testes LSD, “Student-Newman-Keuls” e Duncan, evidenciaram diferenças significativas entre os tratamentos 1; 3 e 5, representando 3 e 5 resultaram em aumentos de 15,97 e 9,27 \% na energia de deformação, respectivamente, quando comparadas ao tratamento 1 . Os tratamentos 2 , 3 e 4 não se diferenciaram, depreendendo-se que a adição de lignossulfonato até $1,5 \%$ afeta a resistência do extrusado. No entanto, com o aumento para $2 \%$, a concentração de lignossulfonato teve um decréscimo no valor médio da resistência. Isso pode ser um indicativo de que a proporção entre aglomerado e aglomerante tenha tornado a mistura menos resistente.

TABELA 6. Valores médios do índice de resistência(J) à compressão, para formulação 1, com respectivos desvios-padrão (D.P.) e coeficientes de variação (C.V.). Average, standard deviation (D.P.) and coefficient of variation (C.V.) of compression resistance index $(\mathrm{J})$, for formulation 1.

\begin{tabular}{cccccc}
\hline & \multicolumn{5}{c}{ Tratamento } \\
& \multicolumn{1}{c}{1} & \multicolumn{1}{c}{2} & \multicolumn{1}{c}{4} & \multicolumn{1}{c}{5} \\
\hline Média (J)* & $0,2884 A^{* *}$ & $0,3296 \mathrm{~B}$ & $0,3345 \mathrm{~B}$ & $0,3230 \mathrm{BC}$ & $0,3152 \mathrm{C}$ \\
D.P. (J) & 0,0649 & 0,0645 & 0,0557 & 0,0576 & 0,0632 \\
C.V. (\%) & 22,59 & 19,59 & 16,66 & 17,81 & 20,06 \\
\hline
\end{tabular}

*para 100 repetições; ** letras distintas indicam diferença significativa entre os valores $(\mathrm{p}<0,05)$. 
Diferentemente dos resultados do ensaio de penetração, a resistência do extrusado aumentou quando determinada pela sua energia de deformação entre pratos planos e paralelos. Esse ensaio mostrou-se mais sensível que o de penetração, sugerindo ser mais adequado para avaliar mecanicamente os extrusados. Apresentou também valor médio de 19,37\% para o coeficiente de variação, muito próximo do obtido para o ensaio de penetração, expressando também variabilidade aceitável para tomada de decisões.

\section{Durabilidade dos extrusados}

A realização de ensaio de durabilidade para produtos extrusados não é uma prática difundida entre as empresas de alimentos para animais, as quais o utilizam para produtos peletizados. Diferentemente, a extrusão promove melhor formatação e agregação das partículas do que a peletização, por utilizar granulometria menor e mais homogênea e, também, por utilizar pressões mais elevadas e maior quantidade de calor.

O resultado dos ensaios de compressão, entre pratos planos rígidos e paralelos, aponta, devido à maior resistência obtida com a adição do lignossulfonato, para um possível ganho na durabilidade. No entanto, os ensaios de durabilidade (Tabela 7) para os vários tratamentos considerados não apresentaram diferença significativa (ANOVA, $\mathrm{p}<0,05$ ), indicando que, para as concentrações consideradas, a inclusão de lignossulfonato de cálcio e magnésio não afeta a durabilidade do extrusado. O valor médio para o índice de durabilidade foi $97,07 \%$, que indica pouca formação de finos. O valor médio de coeficiente de variação, de $0,57 \%$, indica a pequena variação dos resultados e corroboram a robustez da análise.

TABELA 7. Valores médios de durabilidade, para a formulação 1, com desvios-padrão (D.P.) e coeficientes de variação (C.V.), para cada tratamento. Average, standard deviation (D.P.) and coefficient of variation (C.V.) of durability index, for each treatment of formulation 1.

\begin{tabular}{cccccc}
\hline & \multicolumn{5}{c}{ Tratamento } \\
& 1 & 2 & 3 & 4 & 5 \\
\hline Média (\%) & 97,14 & 97,02 & 97,00 & 97,21 & 96,99 \\
D.P. (\%) & 0,9550 & 0,4514 & 0,4514 & 0,4975 & 0,3077 \\
C.V. (\%) & 0,98 & 0,47 & 0,30 & 0,51 & 0,32 \\
\hline
\end{tabular}

Para rações peletizadas para perus, ROBERSON (2003) obteve o valor médio de durabilidade de $82 \%$ para o tratamento-controle, o qual não diferiu significativamente daquele quando à formulação foi adicionado $0,5 \%$ de lignossulfonato. No entanto, o efeito de igual concentração foi significativo (Tabela 6) para o aumento da resistência do extrusado, na formulação considerada. Um incremento relativo na quantidade de lignossulfonato, para $1,25 \%$, resultou num aumento significativo do índice de durabilidade, $67,1 \%$, para peletes destinados a frangos de corte, e apenas 43,1\% para o tratamento-testemunha sem o aditivo (ACAR et al., 1991). No entanto, para extrusados, os valores do índice de durabilidade foram superiores, variando entre 65,65 a 98,99\%, mas com ocorrência mais comum acima de 80\% (FEROUZ, 2013; SITAULA, 2011; UMAR \& RAMEZANI-FARD, 2012). Esses resultados indicam que a durabilidade do pelete é mais afetada pela presença do lignossulfonato que a do extrusado. Note-se que as transformações neste último, devido à injeção de vapor e aumento de temperatura, resultam em ligações mais fortes entre as partículas.

\section{Consumo de energia elétrica}

Os valores de tempo de processamento de 37; 36; 35; 34; 33 e 31 minutos, para os tratamentos de 1 a 6 , respectivamente, apresentam relação linear com os valores das concentrações de lignossulfonato de cálcio e magnésio na mistura (Figura 3). Essa relação é expressa pela equação $\mathrm{Y}=36,5833$ - 1,5X, em que $\mathrm{Y}$ representa o tempo (min) e $\mathrm{X}$ a concentração de lignossulfonato (\%), 
com $\mathrm{R}^{2}=96,43 \%$ e coeficiente de correlação de $-0,9820(\mathrm{p}<0,01)$. Assim, a redução do tempo de processamento implica aumento da produtividade, em concordância com resultados encontrados para peletização, por ZIGGERS (2004), que relatou aumento superior a $20 \%$ na capacidade operacional, quando da adição de aglutinante às rações, explicado como ação lubrificante.

Na Figura 3, são mostradas, também, as variações dos valores médios medidos da potência $(\mathrm{kW})$ e a energia elétrica média $(\mathrm{kWh})$ consumidas durante o processamento da formulação 2.

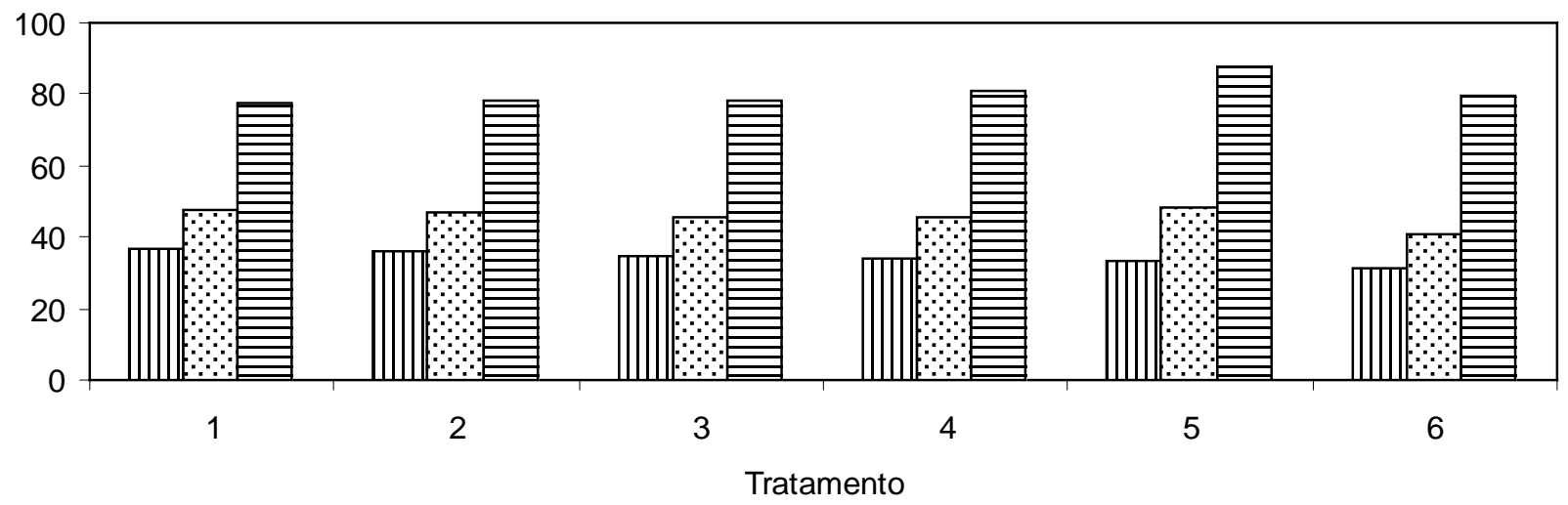

$\square$ tempo de processamento (min) $\quad$ G potência $(\mathrm{kW})$ 目 energia consumida $(\mathrm{kWh})$

FIGURA 3. Valores de tempo de processamento (min.), valores médios da potência (kW) e energia elétrica consumida (kWh) no processamento da formulação 2. Processing time (minute), average power $(\mathrm{kW})$ and electrical energy consumption (kWh) during formulation 2 processing.

Os valores médios de potência para os tratamentos de 1 a 6 foram 47,40; 46,85; 45,26; 45,60; 48,01 e 40,72 kW, respectivamente, com média geral de 45,64 kW e coeficiente de variação de 5,7\%. Os valores médios para o consumo de energia elétrica foram 77,74; 78,50; 78,23; 81,03; 87,98 e 79,54 kWh, com média geral de 80,51 kWh e coeficiente de variação de 4,8\%. Não foi observada correlação linear significativa entre as variações de potência ou consumo energético e as concentrações de lignossulfonato utilizadas. A capacidade de discriminação dessas grandezas elétricas pode ter sido prejudicada, em parte, devido ao baixo valor da potência média, 45,64 kW, que evidencia a subutilização do motor da extrusora, cuja potência nominal é de 149,2 kW. Sabe-se que a utilização de equipamentos superdimensionados deve ser evitada (KALILAN \& MOREY, 2009), mas por questões econômicas e para oferecer uma diversidade de produtos as empresas comumente investem em uma única extrusora em detrimento de sua eficiência.

\section{CONCLUSÕES}

A adição de lignossulfonato de cálcio e magnésio não é recomendada para a redução da formação de finos, muito embora aumente a resistência do extrusado. No entanto, sua adição reduz o tempo de processamento e, consequentemente, aumenta a capacidade operacional da extrusora.

\section{REFERÊNCIAS}

ACAR, N.; MORAN JR., E. T.; REVINGTON, W. H.; BILGILI, S. F. Effect of improved pellet quality from using a Calcium Lignosulfonate Binder on performance and carcass yield of broilers reared under different marketing schemes. Poultry Science, Champaing, v. 70, p. 1339-1344, 1991.

ASAE. Cubes, pellets, and crumbles: definitions and methods for determining density, durability, and moisture content, S269.4. In: ASAE Standards 1994. $41^{\text {th }}$ ed. St Joseph: American Society of Agricultural Enginiers,1994. p. 449-451. 
CHARNET, R.; FREIRE, C.A.L.; CHARNET, E.M.R.; BONVINO, H. Análise de modelos de regressão linear. Campinas: Editora da Unicamp, 2008. 252 p.

FEROUZ, Y. A.; FALLAH, P.; ROSENTRATER, K. A.; MUTHUKUMARAPPAN, K. Modeling single-screw extrusion processing parameters and resulting extrudate properties of DDGS-Based Nile Tilapia (Oreochromis niloticus) feeds. Journal of Food Research, v. 2, n. 2, 2013.

FERRAZ, A.C.O.; HONÓRIO, S.L. Propriedades físicas e mecânicas de frutas. In: NASCIMENTO, L. M.; DE NEGRI, J. D.; MATOS JUNIOR, D. (org.). Tópicos em qualidade e pós-colheita de frutos. Campinas: Instituto Agronômico e Fundag, 2008. p. 143-154.

FRAIHA, M. Efeitos da temperatura, pressão e taxa de cisalhamento sobre a viabilidade de esporo termodúrico durante a extrusão de alimentos para animais. 2008. 196 f. Tese (Doutorado em Engenharia Agrícola) - Universidade Estadual de Campinas, Campinas, 2008.

FRAIHA, M.; BIAGI, J.D.; FERRAZ, A.C.O.; SVERZUT, C.B. Projeto e construção de reômetro capilar para caracterização de alimentos para animais. Ciência Rural, Santa Maria, v. 40, n. 9, set. 2010.

GONZÁLEZ, R. J.; CAVADA, E. P.; PEÑA, J. V.; TORRES, R. L.; DE GREEF, D. M.; DRAGO, S. R. Extrusion conditions and amylose content affect physicochemical properties of extrudates obtained from brown rice grains. International Journal of Food Science, Oxford, 2013. Disponível em: < http://dx.doi.org/10.1155/2013/584148 >. Acesso em: 10 dez. 2013.

KALIYAN, N.; MOREY, R. V. Factors affecting strength and durability of densified biomass products. Biomass and Bioenergy, Oxford, v. 33, p. 337-359, 2009.

KHITRIN, K. S.; FUKS, S. L.; KHITRIN, S. V.; KAZIENKOV, S. A.; METELEVA, D. S. Lignin utilization options and methods. Russian Journal of General Chemistry, New York, v. 82, n. 5, p.977-984, 2012.

MASKELL, D.; WALKER, P.; HEATH, A. The compressive strength of lignosulphonate stabilized extruded earth masonry units. In: INTERNATIONAL CONFERENCE ONT THE STUDY AND CONSERVATION OF EARTHEN ARCHITECTURE HERITAGE, 11., 2012, Lima. Terra 2012... Disponível em: <http://congreso.pucp.edu.pe/terra2012/>. Acesso em: 10 dez. 2013.

MELBAR PRODUTOS DE LIGNINA. Lignosulfonato: catálogo de produtos. São Paulo, 2000. 12p.

ROBERSON, K. D. Effect of feed texture on growth performance and litter moisture when fed to growing-finishing commercial toms. International Journal of Poultry Science, Pakistan, v. 2, n. 3, p. 183-187, 2003.

ROKEY, G. J.; PLATTNER, B.; SOUZA, E. M. Feed extrusion process description. Revista Brasileira Zootecnia, Viçosa, MG, v. 39, p. 510-518, 2010.

SINDICATO NACIONAL DA INDÚSTRIA DE ALIMENTAÇÃO ANIMAL, SINDIRAÇÕES. Compêndio Brasileiro de Alimentação Animal. São Paulo: Sindirações, 2009. 204 p.

SINDICATO NACIONAL DA INDÚSTRIA DE ALIMENTAÇÃO ANIMAL, SINDIRAÇÕES. Boletim Informativo do Setor, dezembro, 2011. Disponível em :

<http://www.sindiracoes.org.br>. Acessado em: 10 dez. 2013.

SINDICATO NACIONAL DA INDÚSTRIA DE ALIMENTAÇÃO ANIMAL. SINDIRAÇÕES. Boletim Informativo do Setor, maio 2013. Disponível em: <http://www.sindiracoes.org.br>. Acesso em: 10 dez. 2013. 
SITAULA, Y. Effect of starch source, screw configuration, and steam injection on physical quality and color development of extruded fish feed. 2011. 28 f. Thesis. (MSc) - Department of Aquacultural and Animal Sciences, Norwegian University of Life Sciences, Oslo, 2011

STATPOINT TECHNOLOGIES. STATIGRAPHICS Plus for Windows 4.1: Professional Version. Warrenton, 1999.

TARASOV, D.; SHAHI, C.; LEITCH, M. Effect of additives on wood pellet physical and thermal characteristics: a review. Forestry, Oxford, 2013. Disponível em:

<http://dx.doi.org/10.1155/2013/876939 >. Acesso em: 10 dez. 2013.

TERRA, P. Líquidos na mistura, peletização, pós-peletização e eExtrusão. In: SIMPÓSIO SOBRE TECNOLOGIA DE ADIÇÃO DE LÍQUIDOS EM SISTEMAS SÓLIDOS, 2000, Paulínia, 2000. Anais...

THOMAS, M.; VAN DER POEL, A. F. B. Physical quality of pelleted animal feed 1. Criteria for pellet quality. Animal Feed Science Technology, Amsterdam, v. 61, p. 89-112, 1996.

UMAR, S.; RAMEZANI-FARD, E. Physical properties of extruded aquafeed with a combination of sago and tapioca starches at different moisture contents. Animal Feed Science Technology, Amsterdam, 2013. Disponível em: <http://dx.doi.org/10.1016/j.anifeedsci.2013.03.009 > . Acesso em: 10 dez. 2013.

WU, T.; ABBOTT, J.A. Firmness and force relaxation characteristics of tomatoes stored intact or as slices. Postharvest Biology and Technology, Amsterdam, v. 24, p. 59-68, 2002.

ZIGGERS, D. The better the pellet, the better the performance. Feed Technology, v. 8, n. 6, p. 1821, 2004. 